\title{
Cerebellar cryptococcosis characterized by a space-occupying lesion in an immunocompetent non-HIV patient
}

This article was published in the following Dove Press journal:

Neuropsychiatric Disease and Treatment

19 December 2014

Number of times this article has been viewed

\author{
Bi-Xia Liu',* \\ $\mathrm{Xi-Jian} \mathrm{Dai}^{2,3, *}$ \\ Heng Liư,* \\ Hong-Han Gong ${ }^{3}$ \\ Yi-Xiang J Wang ${ }^{2}$ \\ Lun-Li Zhang' \\ 'Department of Infectious Diseases, \\ The First Affiliated Hospital \\ of Nanchang University, Nanchang, \\ Jiangxi, People's Republic of China; \\ ${ }^{2}$ Department of Imaging and \\ Interventional Radiology, Prince \\ of Wales Hospital, The Chinese \\ University of Hong Kong, Shatin, \\ New Territories, Hong Kong; \\ ${ }^{3}$ Department of Radiology, The First \\ Affiliated Hospital of Nanchang \\ University, Nanchang, Jiangxi, People's \\ Republic of China; ${ }^{4}$ Department \\ of Radiology, Affiliated Hospital \\ of Zunyi Medical University, Guizhou, \\ People's Republic of China \\ *These authors contributed equally \\ to this work
}

\begin{abstract}
Central nervous system (CNS) cryptococcosis is an opportunistic fungal infection that typically occurs in patients with reduced immunological function, such as patients with AIDS, patients receiving organ transplants, or patients receiving corticosteroid and immunosuppressive therapy. CNS cryptococcosis rarely occurs in immunocompetent patients. CNS cryptococcosis is characterized by meningitis and encephalitis and occasionally forms isolated granulomas. Isolated cerebellar cryptococcoma is a rare condition, especially in immunocompetent patients, and the misdiagnosis rate is high. A definite diagnosis must be based on pathology. To raise awareness of this disease, the clinical data of a patient with cryptococcomas in the right side of the cerebellum are reported.
\end{abstract}

Keywords: cryptococcosis, cerebellar, central nervous system, magnetic resonance imaging, immunosuppressive

\section{Introduction Clinical presentation}

A 61-year-old immunocompetent woman presented with a chronic headache for 10 months that had been progressively worsening for 18 days with emesis. She had an irregular headache without relation to body, position, or time. The patient was a peasant with no particular exposure to avian (especially pigeon) feces or woodworking. The headache got worse, with several nonbloody, nonbilious vomiting episodes, and the patient developed an ataxic gait 18 days before hospital admission. A comprehensive physical examination of her body at admission did not show any pertinent findings. There was no evidence of compromised immunological function or signs of localized infection. She could not complete the hand alternating movement test, her right side of finger-nose test was positive, and her heel-knee-tibia test was suspiciously positive, but Romberg's sign was negative. The rest of the physical examination did not present any abnormalities. Blood cell count, urine analysis, and blood biochemistry were normal. HIV antibody test was repeatedly negative. Gram stain and India ink stain of cerebrospinal fluid (CSF) were negative. CSF and blood were negative for cultures. The cryptococcal antigen latex agglutination test of CSF was negative the first time, but it was positive the second time; the titer was 1:32.

\section{Imaging examination}

Department of Infectious Diseases, The First Affiliated Hospital of Nanchang University, No 17, Yongwai Zheng Street, Donghu District, Nanchang City 330006, Jiangxi, People's Republic of China

Tel +86 79l 88692570

Email lunliz@126.com
Abdominal and chest radiographs were negative. A plain magnetic resonance imaging and long $T_{2}$ signal in the right cerebellar hemisphere with no adjacent edema, and the nodule was significantly enhanced as nodular with a thick and annular wall after contrast administration (Figure 1C-E). There was no midline shift, and the compression of (MRI) scan of the head demonstrated a $26 \times 21 \times 16 \mathrm{~mm}^{3}$ nodule as long or equal to $T_{1}$ 

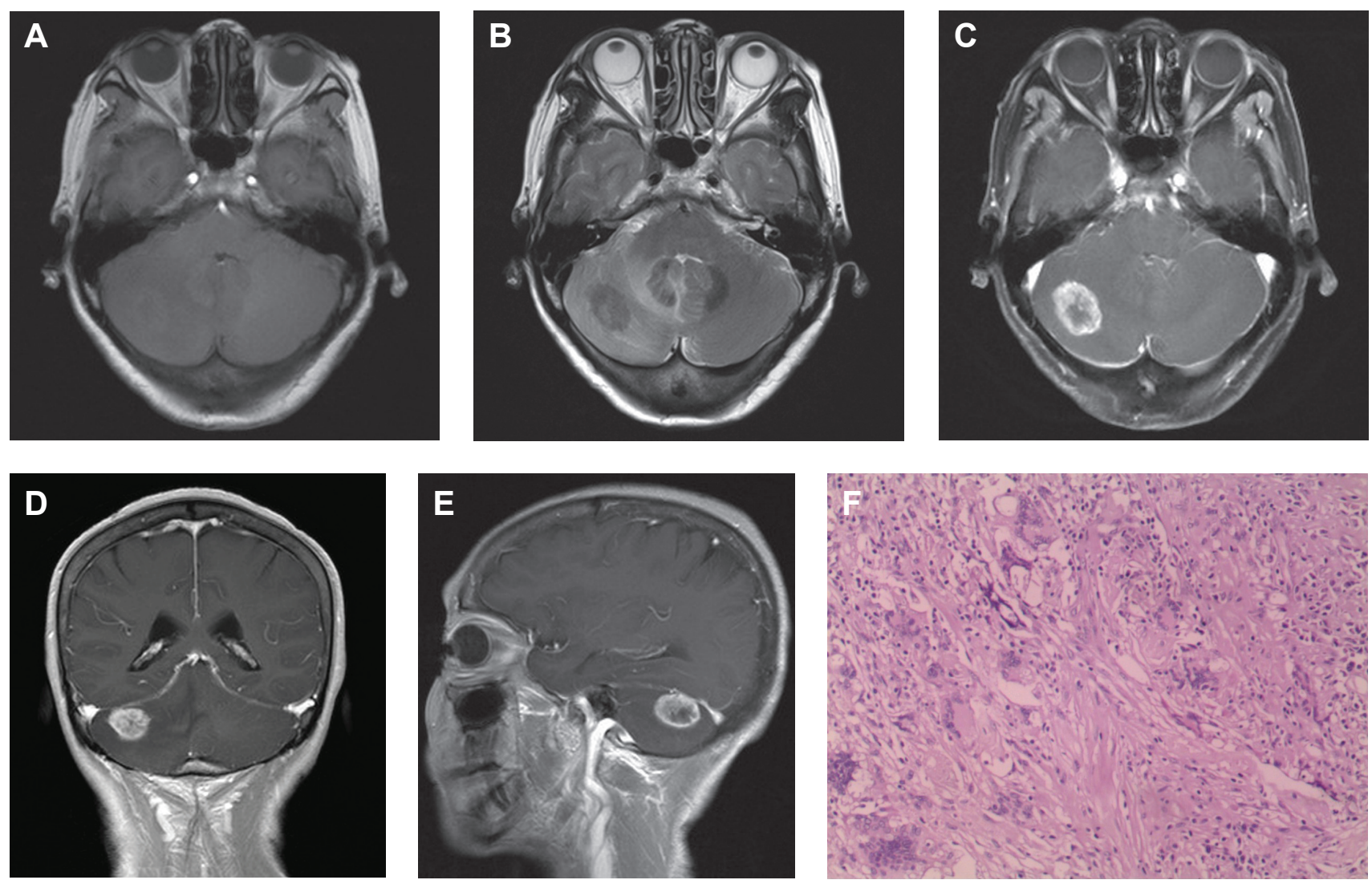

Figure I MRI scans of the head (A-E) and frozen section image (F).

Notes: (A and B) Axial plain MRI scan demonstrates a round mass about $2.6 \times 2.1 \times 1.6 \mathrm{~cm}^{3}$ in the right cerebellar hemisphere. (C-E) Contrast-enhanced MRI scan shows that the right cerebellar mass was enhanced as nodular with a thick and annular wall. (F) Within the tissue frozen section, a large number of mononuclear cells and multinucleated giant cells are diffusely seen. There is a small amount of bacteria circular structure and mesenchyme hyalinization, with a large amount of lymphocyte and little plasma cell infiltration and local tissue necrosis with neutrophils infiltration. Immunohistochemical staining: thallus PAS (+).

Abbreviations: MRI, magnetic resonance imaging; PAS, periodic acid-schiff stain.

adjacent structures was not apparent (Figure 1A and B). The patient was considered to have a metastatic tumor or glioma in the right cerebellar hemisphere.

\section{Operation}

The cerebellar nodule was removed by neurosurgery via a suboccipital craniotomy in the left lateral position. An $80 \mathrm{~mm}$ long straight incision was made $20 \mathrm{~mm}$ outside the right side of the occipital bone of the lower lateral hairline. Intraoperatively, the lower part of the transverse sinus and the surface layer of cerebellum were exposed. Subcortical dissection was performed until a $40 \times 30 \times 20 \mathrm{~mm}$ firm nodule with moderate vascular supply and distinct border was identified, located at the upper portion of the right cerebellar hemisphere. The blood vessels that supply for the nodule were cut off by electrocoagulation, and then en bloc resection of the nodule along the border was performed.

\section{Pathological manifestations}

A portion of the nodule was sent for pathologic analysis. A large number of mononuclear cells and multinucleated giant cells were seen, with diffused distribution. There was a small amount of bacteria circular structure and mesenchyme hyalinization, with a large number of lymphocytes and a few plasma cell infiltrations; local tissue necrosis with neutrophil infiltration was also found in the frozen section tissues (Figure 1F). Immunohistochemical staining yielded CD68 (+), cytokeratin (-), epithelial membrane antigen $(-)$, glial fibrillary acidic protein $(-)$, Ki-67 (-), neurofilament(-), neuron-specific enolase (-), soluble-protein $100(-)$, vimentin (+), cluster of differentiation 1a (-), and thallus periodic acid-schiff stain $(+)$. The neuropathology evidenced a right cerebellar cryptococcal granuloma.

\section{Discussion}

CNS cryptococcosis is a kind of subacute or chronic deep mycosis caused by cryptococcus neoformans. ${ }^{1}$ Cryptococcus neoformans mainly spreads via the respiratory route and infects predilection sites in CNS via hematogenous spread. It usually infects patients with immunosuppression, especially AIDS patients, as well as patients with obvious consumptive disease 
and patients who consumed extensive immunosuppressants and extensive broad-spectrum antibiotic therapy. Occasionally, CNS cryptococcosis infects immunocompetent patients. However, the morbidity of cryptococcosis in immunocompetent patients has increased in recent years. ${ }^{2}$ The main pathologic changes of CNS cryptococcosis were meningitis, meningoencephalitis, vasculitis, and rarely, granulomas. Furthermore, intracranial cryptococcal granulomas were significantly more common in the cerebral hemisphere than in the cerebellar hemisphere. There are two recognized varieties of cryptococcus neoformans: cryptococcus neoformans var neoformans and cryptococcus neoformans var Gattii, which rarely infected immunocompromised patients while usually infecting immunocompetent patients. ${ }^{3,4}$ Cerebral cryptococcoma is the most common form of expression in immunocompetent individuals. ${ }^{5,6}$ There are only a few cases of isolated cerebellar cryptococcoma that were reported in immunocompetent patients. ${ }^{7-10}$

The clinical feature of CNS cryptococcosis is highly variable and nonspecific, which is related to immune status and the types of cryptococcus. ${ }^{11}$ Because of its insidious onset, progressive intracranial hypertension may be the initial symptom, characterized by headache, nausea, and vomiting, and then followed with other symptoms, such as cranial nerve damage and focal neurological signs. Immunocompetent patients may be characterized by meningitis and focal cryptococcomas.

The imaging diagnosis of immunocompetent patients with cerebellar cryptococcomas is a challenge. In general, $\mathrm{CSF}$, including pressure measurement, routine tests, biochemistry indicators, and examination of ink stain of CSF, is essential to the diagnosis. Cryptococcal antigens latex agglutination test of cerebrospinal fluid and blood examination contribute to the diagnosis, but the definitive diagnosis of cryptococcosis must be confirmed by histopathologic examination. In the current patient, the diagnosis of cerebellar cryptococcoma was confirmed by histopathology, whereas the CSF and cryptococcal antigen latex agglutination tests were both negative.

Different sites, pathological types, and stages of CNS cryptococcosis may show different computed tomographic (CT) and MRI findings. ${ }^{12}$ Early stages of cryptococcosis may not show any abnormal CT findings, but a few nonspecific manifestations, including diffuse cerebral atrophy, hydrocephalus, and diffuse cerebral edema, can be found., ${ }^{3,13}$ Cryptococcoma can appear as a localized tumor-like mass when the fungus has involved brain parenchyma. Cryptococcus tumors typically show long $T_{1}$ and long $T_{2}$ signals on MRI, as well as circular enhancement. ${ }^{14}$ Although certain characteristic imaging findings have been described, the differential diagnosis is still difficult because of the overlap of imaging findings with granulomatous tumors and abscesses, especially the tuberculous granuloma.

Early diagnosis and early initiation of treatment are associated with a good prognosis. However, CNS cryptococcosis can be fatal if not treated. The therapeutic goal is to control the initial infection and lifelong antifungal therapy for patients with HIV coinfection and to cure the cryptococcus infection completely for patients without HIV coinfection. CSF should be sampled to assess the therapeutic effect after initial therapy for 2 weeks. ${ }^{7}$

\section{Conclusion}

The lack of specificity of clinical manifestations and the low detection rate of cryptococcus in CSF bring difficulties to its diagnosis. In this reported case, CNS cryptococcosis manifested as an intracranial lesion, which was misdiagnosed as a tuberculoma or a cerebral tumor. It is necessary to perform multiple smears and cultures in CSF examination. Radiological examination, especially by MRI, may make a suggestive diagnosis. A definite diagnosis is confirmed by histological examination.

\section{Acknowledgments}

This work was supported by Jiangxi Provincial Department of Graduate Innovation Foundation (Grants YC2013-S007 and YC2013-S011), Chinese Department of National Innovation Experiment Program for University Students (Grants 201210403052 and 2012181), and Jiangxi Provincial Department of Science and Technology support program (Grant 20141BBG70026).

\section{Disclosure}

The authors report no conflicts of interest in this work.

\section{References}

1. Perfect JR. The triple threat of cryptococcosis: it's the body site, the strain, and/or the host. MBio. 2012;3(4):e00165-e12.

2. Desalermos A, Kourkoumpetis TK, Mylonakis E. Update on the epidemiology and management of cryptococcal meningitis. Expert Opin Pharmacother. 2012;13(6):783-789.

3. Correa MP, Severo LC, Oliveira FM, Irion K, Londero AT. The spectrum of computerized tomography (CT) findings in central nervous system (CNS) infection due to Cryptococcus neoformans var. gattii in immunocompetent children. Rev Inst Med Trop Sao Paulo. 2002;44(5): 283-287.

4. Mitchell DH, Sorrell TC, Allworth AM, et al. Cryptococcal disease of the CNS in immunocompetent hosts: influence of cryptococcal variety on clinical manifestations and outcome. Clin Infect Dis. 1995;20(3): 611-616.

5. Kesler R, Maertens P. Pontine cryptococcoma in a nonimmunocompromised individual: MRI characteristics. J Neuroimaging. 1999;9(2) $118-121$. 
6. Li Q, You C, Liu Q, Liu Y. Central nervous system cryptococcoma in immunocompetent patients: a short review illustrated by a new case. Acta Neurochir (Wien). 2010;152(1):129-136.

7. Gologorsky Y, DeLaMora P, Souweidane MM, Greenfield JP. Cerebellar cryptococcoma in an immunocompetent child. Case report. J Neurosurg. 2007;107(4)(suppl):314-317.

8. Kanaly CW, Selznick LA, Cummings TJ, Adamson DC. Cerebellar cryptococcoma in a patient with undiagnosed sarcoidosis: case report. Neurosurgery. 2007;60(3):E571.

9. Reis F, Schwingel R, Souza TF, Queiroz LS, Zanardi VA. Cerebellar cryptococcoma in a non-HIV patient. Arq Neuropsiquiatr. 2011;69(5):851.

10. Nakwan N, Songjamrat A, Tungsinmonkong K, Nakwan N. Cerebellar cryptococcoma in an immunocompetent adult patient. Southeast Asian J Trop Med Public Health. 2009;40(5):1034-1037.
11. Peachey PR, Gubbins PO, Martin RE. The association between cryptococcal variety and immunocompetent and immunocompromised hosts. Pharmacotherapy. 1998;18(2):255-264.

12. Scozzafava J, Block H, Asdaghi N, Siddiqi ZA. Teaching NeuroImage: cryptococcal brain pseudocysts in an immunocompetent patient. Neurology. 2007;69(9):E6-E7.

13. Awasthi M, Patankar T, Shah P, Castillo M. Cerebral cryptococcosis: atypical appearances on CT. Br J Radiol. 2001;74(877):83-85.

14. Ho TL, Lee HJ, Lee KW, Chen WL. Diffusion-weighted and conventional magnetic resonance imaging in cerebral cryptococcoma. Acta Radiol. 2005;46(4):411-414.

\section{Publish your work in this journal}

Neuropsychiatric Disease and Treatment is an international, peerreviewed journal of clinical therapeutics and pharmacology focusing on concise rapid reporting of clinical or pre-clinical studies on a range of neuropsychiatric and neurological disorders. This journal is indexed on PubMed Central, the 'PsycINFO' database and CAS, and is the official journal of The International Neuropsychiatric Association (INA). The manuscript management system is completely online and includes a very quick and fair peer-review system, which is all easy to use. Visit http://www.dovepress.com/testimonials.php to read real quotes from published authors.

Submit your manuscript here: http://www.dovepress.com/neuropsychiatric-disease-and-treatment-journal 